\title{
Atomic structure, radiative lifetime and oscillator strength calculations in doubly ionized molybdenum (MoIII)
}

\author{
Pascal Quinet \\ Astrophysique et Spectroscopie, Université de Mons, B-7000 Mons, Belgium \\ IPNAS, Université de Liège, B15 Sart Tilman, B-4000 Liège, Belgium \\ E-mail: quinet@umons.ac.be
}

Received 11 June 2014, revised 15 September 2014

Accepted for publication 23 September 2014

Published DD MM 2014

\begin{abstract}
Radiative lifetimes, transition probabilities and oscillator strengths in doubly ionized molybdenum (Mo III) are reported for the first time in the present paper. This new set of atomic data has been obtained by using a semi-empirical computational technique based on the pseudorelativistic Hartree-Fock approach in which a large amount of intravalence and core-valence electron correlations were included. In view of the lack of theoretical and experimental data available in the literature for this ion, the reliability of the results obtained in our work is discussed on the basis of comparisons with the isoelectronic ion $\mathrm{Nb}$ II for which an investigation was recently performed using a similar method (Nilsson et al 2010 Astron. Astrophys. 511 A16).
\end{abstract}

S Online supplementary data available from stacks.iop.org/ps/0/000000/mmedia

Keywords: atomic structure, radiative rates, Hartree-Fock calculations

\section{Introduction}

Molybdenum, the forty-second chemical element of the periodic table $(Z=42)$ has many applications in different scientific fields. For example, in astrophysics, the abundance of molybdenum in some metal-poor stars was found to be extremely enhanced, as high or higher than the neighboring even- $Z$ elements ruthenium and zirconium (Peterson 2011,2013 ) constraining the possible nucleosynthesis scenarios envisioned for the production of nuclei in this mass range. In fusion research, molybdenum is used as component of plasma-facing material in different devices such as Alcator C-Mod reactor (Lipschultz et al 2006) or the experimental advanced superconducting tokamak, EAST (Liu et al 2013). These applications require a large number of spectroscopic parameters characterizing the different ionization degrees of molybdenum but so far, unfortunately, for lowly charged states, radiative data were only published for the first two spectra.

More precisely, in Mo I, relative transition probabilities for some lines were measured by Dickerman and Deuel (1964) using a high current argon arc plasma mixed with molybdenum in dust form. The lifetimes of some levels of the $4 d^{5} 5 p$ and $4 d^{4} 5 s 5 p$ configurations were determined by zerofield level-crossing technique by Baumann et al (1978). These measurements were then extended to additional levels by Duquette et al (1981) and Kwiatkowski et al (1981) with selective laser excitation and time-resolved observation of the reemitted fluorescence. Oscillator strengths of $174 \mathrm{Mo}$ I lines in the range $2470-5570 \AA$ were obtained by Schnehage et al (1983) from wall-stabilized arc and hollow cathode measurements. The radiative lifetimes of 56 and 14 excited levels were respectively measured by Whaling $(1984,1986)$ using time-resolved laser fluorescence spectroscopy. Emission branching ratios for the decay of these levels were measured to determine absolute transition probabilities for a total of about 700 lines in the wavelength range 2600-9767 A. Decay rates for 2835 Mo I lines between 2548 and $10565 \AA$ were also published by Whaling and Brault (1988) who combined level lifetimes, excited level populations measured in an inductively coupled plasma (ICP) source, and emission branching ratios measured with the ICP source and with a hollow cathode discharge source. Later, the fine structure and transition probabilities were studied by Palmeri and Wyart 
(1998) in the semi-empirical Racah-Slater framework by means of the relativistic Hartree-Fock (HFR) and fitting methods developed by Cowan (1981). Finally, radiative lifetimes for 14 odd-parity levels with the energy range between 31654.79 and $47184.52 \mathrm{~cm}^{-1}$ of Mo I were measured by Jiang et al (2013) using the time-resolved laser-induced fluorescence technique. Branching fraction measurements of these levels were performed based on the emission spectrum of a hollow cathode lamp. By combining the measured lifetimes and branching fractions, new absolute transition probabilities and oscillator strengths for 130 transitions in the wavelength range extending from 2754 to $6005 \AA$ were derived.

In the case of Mo II, Hannaford and Lowe (1983) measured lifetimes for 15 levels using the laser induced fluorescence technique applied to a sputtered metal vapour. Later on, Sikström et al (2001) reported experimental radiative lifetimes for 10 levels by the same method. With the HFR approach including core-polarization effects, theoretical lifetimes for 37 levels of Mo II and the oscillator strengths of the depopulating transitions were calculated by Quinet (2002). More recently, Lundberg et al (2010) measured new radiative lifetimes for 14 odd levels in the energy range $48000-61000 \mathrm{~cm}^{-1}$ while Jiang et al (2012) reported experimental values for 13 odd levels between 48022 and $63497 \mathrm{~cm}^{-1}$. In these two latter works, new transition probabilities were also obtained using the HFR method.

To our best knowledge, no experimental neither theoretical radiative parameters have been published so far for Mo III. In order to fill in this gap, in the present paper, we report on calculations of oscillator strengths and transition probabilities in this ion performed using the HFR approach including core-polarization effects. This work is an extension of our recent investigations of the fifth row elements $\mathrm{Rb}$ III (Zhang et al 2014), Y II, Y III (Biémont et al 2011), Zr II (Malcheva et al 2006), Nb I (Malcheva et al 2011), Nb II, Nb III (Nilsson et al 2010), Mo II (Quinet 2002, Lundberg et al 2010, Jiang et al 2012), Tc II (Palmeri et al 2007), Ru I (Fivet et al 2009), Ru II, Ru III (Palmeri et al 2009), Rh II (Quinet et al 2011, 2012), Rh III (Zhang et al 2013a), Pd I (Xu et al 2006), Pd III (Zhang et al 2013a), Ag II (Biémont et al 2005, Campos et al 2005), Ag III (Zhang et al 2013a), Sn I (Zhang et al 2008, 2009, 2010), Sb I (Hartman et al 2010), Te II and Te III (Zhang et al 2013b).

\section{The Mo III spectrum}

The most recent and complete analysis of the Mo III spectrum was published by Iglesias et al (1990) who classified approximately 3100 lines in the range 800-2100 A, extending in this way their previous data of 679 lines covering the range 1100-3250 A (Iglesias et al 1988). These observations were performed under similar conditions using molybdenum spectra produced in a sliding spark discharge and recorded photographically on the NIST (National Institute of Standards and Technology) $10.7 \mathrm{~m}$ normal-incidence vacuum spectrograph equipped with a $12001 \mathrm{~mm}^{-1}$ grating blazed at
$1200 \AA$. The wavelength uncertainty of the observed lines was estimated to be $\pm 0.005 \AA$. Altogether both investigations led to the establishment of 149 energy levels in the $4 d^{4}$, $4 d^{3} 5 s, 4 d^{2} 5 s^{2}, 4 d^{3} 5 d$ and $4 d^{3} 6 s$ even configurations and 181 energy levels in the $4 d^{3} 5 p$ and $4 d^{2} 5 s 5 p$ odd configurations. According to Iglesias et al (1990), the uncertainties of the energy level values listed in their tables are generally less than $\pm 0.10 \mathrm{~cm}^{-1}$ and no greater than $\pm 0.20 \mathrm{~cm}^{-1}$. Semiempirical HFR calculations were also carried out by the same authors for each of the following rather limited interacting configuration groups : (1) $4 d^{4}+4 d^{3} 5 s+4 d^{2} 5 s^{2}$, (2) $4 d^{3} 5 d+$ $4 d^{3} 6 s$, and (3) $4 d^{3} 5 p+4 d^{2} 5 s 5 p$. This allowed them to give LS designations to the experimental levels with average purities of $83 \%, 59 \%$ and $63 \%$ for the three groups of configurations mentioned above, respectively.

\section{Atomic structure calculations}

\subsection{Pseudo-HFR model}

The pseudo-HFR approach described by Cowan (1981) was used for modeling the atomic structure and calculating the radiative parameters in Mo III. The interacting configurations explicitly included in the physical model were exactly the same as those considered in our recent study related to the isoelectronic ion $\mathrm{Nb}$ II (Nilsson et al 2010), i.e. $4 d^{4}+4 d^{3} 5 s+$ $4 \mathrm{~d}^{3} 6 \mathrm{~s}+4 \mathrm{~d}^{3} 5 \mathrm{~d}+4 \mathrm{~d}^{2} 5 \mathrm{~s}^{2}+4 \mathrm{~d}^{2} 5 \mathrm{p}^{2}+4 \mathrm{~d}^{2} 5 \mathrm{~d}^{2}+4 \mathrm{~d}^{2} 6 \mathrm{~s}^{2}+$ $4 d^{2} 5 s 6 s+4 d^{2} 5 s 5 d+4 d^{2} 4 f 5 p+4 d^{2} 5 p 5 f+4 d^{2} 5 d 6 s+4 d^{2} 5 p 6 p$ for the even parity and $4 d^{3} 5 p+4 d^{3} 6 p+4 d^{3} 4 f+4 d^{3} 5 f+$ $4 d^{2} 5 s 5 p+4 d^{2} 5 s 6 p+4 d^{2} 4 f 5 s+4 d^{2} 4 f 5 d+4 d^{2} 5 s 5 f+4 d^{2} 5 p 6 s$ $+4 d^{2} 5 p 5 d+4 d^{2} 6 s 6 p$ for the odd parity. The relativistic corrections were the mass-velocity and the one-body Darwin terms, as well as the Blume-Watson spin-orbit interaction. The latter contribution includes the part of the Breit interaction that can be reduced to a one-body operator.

\subsection{Core-polarization effects}

Core-valence interactions were taken into account using a polarization model potential and a correction to the dipole operator following a well-established procedure giving rise to the HFR + CPOL method (see e.g. Quinet et al 1999, 2002). In the present work, similarly to our previous work on $\mathrm{Nb}$ II ion (Nilsson et al 2010), the polarization model adopted for Mo III was based on a $\mathrm{Mo}^{4+}$ ionic core surrounded by two valence electrons. In this model, the CPOL effects were thus included using the dipole polarizability of Mo v given by Fraga et al (1976), i.e. $\alpha_{\mathrm{d}}=3.71 \mathrm{a}_{0}^{3}$ while the cut-off radius was chosen to be equal to $r_{c}=1.60 a_{0}$ which corresponds to the mean value $\langle r\rangle$ of the outermost $4 d$ core orbital computed with the HFR Cowan's code.

\subsection{Semi-empirical optimization of radial parameters}

The HFR + CPOL method was then combined with a leastsquares optimization routine that minimize the discrepancies between calculated and experimental energy levels published by Iglesias et al (1990). In the even parity, all the 149 
experimentally known levels were fitted using, as adjustable parameters, the average energies, the electrostatic interaction integrals, and the spin-orbit parameters corresponding to the $4 d^{4}, 4 d^{3} 5 s, 4 d^{3} 6 s, 4 d^{3} 5 d$ and $4 d^{2} 5 s^{2}$ configurations. In the case of odd-parity levels, the 159 experimental values below $143000 \mathrm{~cm}^{-1}$ were included in the fitting procedure using the radial parameters of the $4 d^{3} 5 p$ and $4 d^{2} 5 s 5 p$ configurations as variable parameters. The levels situated above $143000 \mathrm{~cm}^{-1}$ were excluded from the semi-empirical adjustment because it was found that many of those might be expected to overlap unknown levels belonging to higher configurations such as $4 d^{3} 6 p$ and $4 d^{3} 4 f$, these two latter configurations being predicted to start around 144000 and $149000 \mathrm{~cm}^{-1}$, respectively, in our calculations. The mean deviations, $\Delta E$, obtained when fitting the levels were found to be equal to 65 and $81 \mathrm{~cm}^{-1}$ for even and odd parities, respectively. It was also found that LScoupling was quite satisfactory for characterizing most of the levels considered in the present work, the average LS purities being calculated equal to $75 \%$ for the 149 levels of even parity and to $60 \%$ for the 159 levels of the odd parity. This confirms the results obtained previously by Iglesias et al (1990) using very limited theoretical models. The full lists of energy levels are given as supplementary files in table $\mathrm{S} 1$ and S 2 for even and odd parities, respectively (available from ...).

\section{Results and discussion}

\subsection{Radiative lifetimes}

Radiative lifetimes obtained in the present work are reported in table 1 for energy levels belonging to the $4 d^{3} 5 d, 4 d^{3} 6 s$ even-parity configurations and in table 2 for energy levels of the $4 d^{3} 5 p, 4 d^{2} 5 s 5 p$ odd-parity configurations. Unfortunately, no experimental neither theoretical values in Mo III were previously published in the literature for comparison. However, an argument for assessing the reliability of the present results can be obtained from isoelectronic comparisons, particularly from results obtained recently in $\mathrm{Nb}$ II (Nilsson et al 2010), the HFR + CPOL model adopted in this work being the same as that chosen for this isoelectronic ion. More precisely, in this latter work, radiative lifetimes of 17 states belonging to the $4 d^{3} 5 p$ configuration in $\mathrm{Nb}$ II were measured using the time-resolved laser-induced fluorescence technique. The comparison of these accurate laboratory measurements with the HFR + CPOL calculations showed that the computed values were in excellent agreement (within 10\%) with experimental lifetimes. An excellent agreement (within a few $\%$ ) was also found when comparing the calculations with the experimental laser spectroscopy measurements obtained by Salih and Lawler (1983) for seven $4 d^{3} 5 p$ levels of $\mathrm{Nb}$ II. Consequently, a similar accuracy can also be expected for most of the radiative lifetimes obtained in the present work for Mo III.
Table 1. Calculated radiative lifetimes for levels belonging to the even-parity configurations of Mo III.

\begin{tabular}{|c|c|c|c|}
\hline Level & $\begin{array}{l}E_{\exp }^{a} \\
\left(\mathrm{~cm}^{-1}\right)\end{array}$ & $\begin{array}{l}E_{\text {calc }}{ }^{-1} \\
\left(\mathrm{~cm}^{-1}\right)\end{array}$ & $\begin{array}{r}\text { Lifetime }^{\mathrm{b}} \\
(\mathrm{ns})\end{array}$ \\
\hline $4 d^{3}\left({ }^{4} F\right) 5 d{ }^{5} H_{3}$ & 130365.61 & 130354 & 0.78 \\
\hline $4 d^{3}\left({ }^{4} F\right) 5 d^{3} D_{1}$ & 130629.3 & 130610 & 0.87 \\
\hline $4 d^{3}\left({ }^{4} F\right) 5 d^{5} P_{1}$ & 130886.95 & 130859 & 0.87 \\
\hline $4 d^{3}\left({ }^{4} F\right) 5 d{ }^{5} \mathrm{H}_{4}$ & 130918.16 & 130898 & 0.80 \\
\hline $4 d^{3}\left({ }^{4} F\right) 5 d^{5} G_{2}$ & 131072.5 & 131061 & 0.85 \\
\hline $4 d^{3}\left({ }^{4} F\right) 5 d^{5} P_{2}$ & 131379.2 & 131359 & 0.88 \\
\hline $4 d^{3}\left({ }^{4} F\right) 6 s{ }^{5} F_{1}$ & 131396.0 & 131435 & 0.90 \\
\hline $4 d^{3}\left({ }^{4} F\right) 5 d{ }^{5} G_{3}$ & 131592.6 & 131566 & 0.87 \\
\hline $4 d^{3}\left({ }^{4} F\right) 5 d{ }^{5} H_{5}$ & 131607.85 & 131582 & 0.82 \\
\hline $4 d^{3}\left({ }^{4} F\right) 6 s{ }^{5} F_{2}$ & 131647.45 & 131639 & 0.85 \\
\hline $4 d^{3}\left({ }^{4} F\right) 5 d^{5} F_{1}$ & 131900.8 & 131901 & 0.91 \\
\hline $4 d^{3}\left({ }^{4} F\right) 5 d^{3} D_{2}$ & 131913.3 & 131928 & 0.91 \\
\hline $4 d^{3}\left({ }^{4} F\right) 5 d^{5} G_{4}$ & 132173.9 & 132145 & 0.89 \\
\hline $4 d^{3}\left({ }^{4} F\right) 5 d^{5} F_{2}$ & 132228.4 & 132225 & 0.91 \\
\hline $4 d^{3}\left({ }^{4} F\right) 6 s{ }^{5} F_{3}$ & 132279.6 & 132252 & 0.86 \\
\hline $4 d^{3}\left({ }^{4} F\right) 5 d^{5} P_{3}$ & 132337.96 & 132342 & 0.86 \\
\hline $4 d^{3}\left({ }^{4} F\right) 5 d{ }^{5} H_{6}$ & 132424.4 & 132396 & 0.84 \\
\hline $4 d^{3}\left({ }^{4} F\right) 5 d{ }^{5} F_{3}$ & 132666.7 & 132661 & 0.88 \\
\hline $4 d^{3}\left({ }^{4} F\right) 5 d^{5} G_{5}$ & 132951.3 & 132920 & 0.89 \\
\hline $4 d^{3}\left({ }^{4} F\right) 5 d^{5} F_{4}$ & 133034.7 & 132974 & 0.86 \\
\hline $4 d^{3}\left({ }^{4} F\right) 5 d^{3} D_{3}$ & 133151.83 & 133137 & 0.93 \\
\hline $4 d^{3}\left({ }^{4} \mathrm{~F}\right) 5 \mathrm{~d}^{5} \mathrm{H}_{7}$ & 133337.7 & 133307 & 0.85 \\
\hline $4 d^{3}\left({ }^{4} F\right) 6 s{ }^{5} F_{4}$ & 133446.5 & 133381 & 0.89 \\
\hline $4 d^{3}\left({ }^{4} F\right) 5 d^{3} P_{0}$ & 133508.9 & 133511 & 0.86 \\
\hline $4 d^{3}\left({ }^{4} F\right) 6 s{ }^{3} F_{2}$ & 133563.4 & 133636 & 0.89 \\
\hline $4 d^{3}\left({ }^{4} F\right) 5 d^{3} \mathrm{H}_{4}$ & 133739.17 & 133703 & 0.92 \\
\hline $4 d^{3}\left({ }^{4} F\right) 5 d{ }^{5} F_{5}$ & 133782.3 & 133722 & 0.85 \\
\hline $4 d^{3}\left({ }^{4} F\right) 5 d^{5} G_{6}$ & 134010.4 & 133979 & 0.90 \\
\hline $4 d^{3}\left({ }^{4} F\right) 5 d^{3} P_{1}$ & 134185.0 & 134172 & 0.88 \\
\hline $4 d^{3}\left({ }^{4} F\right) 5 d^{3} G_{3}$ & 134295.5 & 134298 & 0.93 \\
\hline $4 d^{3}\left({ }^{4} F\right) 6 s{ }^{5} F_{5}$ & 134371.48 & 134205 & 0.91 \\
\hline $4 d^{3}\left({ }^{4} F\right) 6 s{ }^{3} F_{3}$ & 134665.06 & 134631 & 0.90 \\
\hline $4 d^{3}\left({ }^{4} F\right) 5 d^{3} H_{5}$ & 134799.5 & 134775 & 0.91 \\
\hline $4 d^{3}\left({ }^{4} F\right) 5 d^{3} F_{2}$ & 135112.06 & 135128 & 0.95 \\
\hline $4 d^{3}\left({ }^{4} F\right) 5 d^{3} G_{4}$ & 135261.56 & 135276 & 0.91 \\
\hline $4 d^{3}\left({ }^{4} F\right) 5 d^{3} P_{2}$ & 135441.05 & 135443 & 0.92 \\
\hline $4 d^{3}\left({ }^{4} F\right) 6 s{ }^{3} F_{4}$ & 135857.46 & 135715 & 0.90 \\
\hline $4 d^{3}\left({ }^{4} F\right) 5 d^{3} F_{3}$ & 135882.9 & 135897 & 0.96 \\
\hline $4 d^{3}\left({ }^{4} F\right) 5 d^{3} \mathrm{H}_{6}$ & 135979.5 & 135965 & 0.90 \\
\hline $4 d^{3}\left({ }^{4} F\right) 5 d^{3} G_{5}$ & 136391.56 & 136422 & 0.92 \\
\hline $4 d^{3}\left({ }^{4} F\right) 5 d^{3} F_{4}$ & 136574.54 & 136599 & 0.95 \\
\hline $4 d^{3}\left({ }^{2} G\right) 5 d^{1} F_{3}$ & 141993.9 & 142033 & 0.87 \\
\hline $4 d^{3}\left({ }^{2} G\right) 5 d^{3} H_{4}$ & 142696.6 & 142707 & 0.81 \\
\hline $4 d^{3}\left({ }^{2} G\right) 5 d^{1} H_{5}$ & 142712.95 & 142735 & 0.89 \\
\hline $4 d^{3}\left({ }^{2} G\right) 5 d^{3} I_{6}$ & 142822.32 & 142876 & 0.84 \\
\hline $4 d^{3}\left({ }^{2} G\right) 5 d^{3} H_{5}$ & 142946.75 & 142950 & 0.85 \\
\hline $4 d^{3}\left({ }^{4} P\right) 5 d^{5} F_{5}$ & 142950.65 & 142975 & 0.90 \\
\hline $4 d^{3}\left({ }^{2} G\right) 5 d^{3} G_{4}$ & 143198.04 & 143219 & 0.88 \\
\hline $4 d^{3}\left({ }^{2} G\right) 6 s{ }^{3} G_{3}$ & 143396.8 & 143051 & 0.90 \\
\hline $4 d^{3}\left({ }^{2} G\right) 5 d^{3} I_{7}$ & 143528.65 & 143537 & 0.88 \\
\hline $4 d^{3}\left({ }^{2} G\right) 6 s^{3} G_{4}$ & 143568.8 & 143450 & 0.88 \\
\hline $4 d^{3}\left({ }^{2} G\right) 5 d^{3} G_{5}$ & 143653.5 & 143705 & 0.87 \\
\hline $4 d^{3}\left({ }^{2} G\right) 5 d^{3} H_{6}$ & 143829.4 & 143830 & 0.86 \\
\hline $4 d^{3}\left({ }^{2} G\right) 6 s{ }^{3} G_{5}$ & 144121.65 & 143907 & 0.91 \\
\hline $4 d^{3}\left({ }^{2} G\right) 6 s{ }^{1} G_{4}$ & 144656.26 & 144495 & 0.91 \\
\hline $4 d^{3}\left({ }^{2} G\right) 5 d^{1} I_{6}$ & 144783.96 & 144741 & 0.87 \\
\hline $4 d^{3}\left({ }^{4} P\right) 5 d^{3} F_{4}$ & 145096.74 & 145098 & 0.91 \\
\hline
\end{tabular}


Table 1. (Continued.)

\begin{tabular}{|c|c|c|c|}
\hline Level & $\begin{array}{l}E_{\exp }{ }^{a} \\
\left(\mathrm{~cm}^{-1}\right)\end{array}$ & $\begin{array}{l}\mathrm{E}_{\mathrm{calc}} \mathrm{b} \\
\left(\mathrm{cm}^{-1}\right)\end{array}$ & $\begin{array}{r}\text { Lifetime }^{\mathrm{b}} \\
\text { (ns) }\end{array}$ \\
\hline $4 d^{3}\left({ }^{2} \mathrm{H}\right) 5 d{ }^{1} \mathrm{H}_{5}$ & 145904.28 & 145927 & 0.89 \\
\hline $4 d^{3}\left({ }^{2} \mathrm{H}\right) 5 \mathrm{~d}^{3} \mathrm{I}_{7}$ & 146257.14 & 146257 & 0.87 \\
\hline $4 d^{3}\left({ }^{2} H\right) 5 d^{3} I_{6}$ & 146277.52 & 146260 & 0.86 \\
\hline $4 d^{3}\left({ }^{2} H\right) 5 d^{3} I_{5}$ & 146342.74 & 146317 & 0.86 \\
\hline $4 d^{3}\left({ }^{2} D\right) 5 d^{3} G_{4}$ & 147431.23 & 147379 & 0.88 \\
\hline $4 d^{3}\left({ }^{2} \mathrm{H}\right) 6 \mathrm{~s}^{3} \mathrm{H}_{4}$ & 147703.6 & 147730 & 0.90 \\
\hline $4 d^{3}\left({ }^{2} \mathrm{H}\right) 6 \mathrm{~s}^{3} \mathrm{H}_{5}$ & 147752.1 & 147814 & 0.90 \\
\hline $4 d^{3}\left({ }^{2} H\right) 5 d^{3} K_{6}$ & 147758.2 & 147753 & 0.83 \\
\hline $4 d^{3}\left({ }^{2} \mathrm{H}\right) 5 \mathrm{~d}^{3} \mathrm{~K}_{7}$ & 147963.3 & 147947 & 0.87 \\
\hline $4 d^{3}\left({ }^{2} \mathrm{H}\right) 6 \mathrm{~s}^{3} \mathrm{H}_{6}$ & 147984.1 & 148082 & 0.90 \\
\hline $4 d^{3}\left({ }^{2} \mathrm{H}\right) 5 d^{1} \mathrm{~K}_{7}$ & 148595.3 & 148584 & 0.92 \\
\hline $4 d^{3}\left({ }^{2} \mathrm{H}\right) 6 \mathrm{~s}{ }^{1} \mathrm{H}_{5}$ & 148816.1 & 148818 & 0.89 \\
\hline $4 d^{3}\left({ }^{2} \mathrm{H}\right) 5 \mathrm{~d}^{3} \mathrm{H}_{5}$ & 151580.2 & 151588 & 0.84 \\
\hline $4 d^{3}\left({ }^{2} F\right) 6 s{ }^{3} F_{4}$ & 156378.82 & 156256 & 0.88 \\
\hline $4 d^{3}\left({ }^{2} F\right) 6 s{ }^{3} F_{3}$ & 156587.8 & 156362 & 0.87 \\
\hline $4 d^{3}\left({ }^{2} F\right) 6 s{ }^{1} F_{3}$ & 157546.6 & 157385 & 0.92 \\
\hline
\end{tabular}

\subsection{Transition rates}

Oscillator strengths and transition probabilities were computed for Mo III spectral lines using our HFR + CPOL model. Due to space limitations, only a small sample of results corresponding to the strongest $g f$-values, i.e. $\log g f>0$, is presented in table 3. This corresponds to 172 lines in the ultraviolet region from 1081 to $2634 \AA$. The full set of data containing transition rates for 7555 lines in the wavelength range from extreme ultraviolet to mid-infrared ( $724 \AA-9.32$ $\mu \mathrm{m})$ is reported in table S 3 given as supplementary file (available from ...). Note that, in those two tables, the wavelengths (given in vacuum below $2000 \AA$ and in air above that limit) were deduced from experimental energy level values published by Iglesias et al (1990). Laboratory measurements of accurate radiative lifetimes and branching fractions in Mo III would now be welcome to definitely assess the accuracy of the new theoretical results obtained in the present work. However, in view of the very good agreement observed when comparing available experimental data with our previous calculations performed in many other similar ions using the same HFR + CPOL approach (see reference quoted in the last paragraph of introduction), one can expect uncertainties of the order of 10-20\% for the computed transition rates listed in the present paper, at least for the most intense lines.

\section{Acknowledgments}

The author is Research Director of the Belgian National Fund for Scientific Research F.R.S.-FNRS. Financial support from this organization is acknowledged.
Table 2. Calculated radiative lifetimes for levels belonging to the odd-parity configurations of Mo III.

\begin{tabular}{|c|c|c|c|}
\hline Level & $\begin{array}{l}E_{\exp }^{a} \\
\left(\mathrm{~cm}^{-1}\right)\end{array}$ & $\begin{array}{l}E_{c a l c}^{b} \\
\left(\mathrm{~cm}^{-1}\right)\end{array}$ & $\begin{array}{c}\text { Lifetime }^{\mathrm{b}} \\
\text { (ns) }\end{array}$ \\
\hline $4 d^{3}\left({ }^{4} F\right) 5 p{ }^{5} G_{2}$ & 73853.18 & 73867 & 3.17 \\
\hline $4 d^{3}\left({ }^{4} F\right) 5 p^{5} G_{3}$ & 74724.72 & 74731 & 3.07 \\
\hline $4 d^{3}\left({ }^{4} F\right) 5 p{ }^{5} G_{4}$ & 75816.51 & 75811 & 2.95 \\
\hline $4 d^{3}\left({ }^{4} F\right) 5 p{ }^{3} D_{1}$ & 75972.36 & 76006 & 1.65 \\
\hline $4 d^{3}\left({ }^{4} F\right) 5 p^{3} D_{2}$ & 76836.82 & 76871 & 1.51 \\
\hline $4 d^{3}\left({ }^{4} F\right) 5 p^{5} G_{5}$ & 77113.28 & 77087 & 2.82 \\
\hline $4 d^{3}\left({ }^{4} F\right) 5 p{ }^{5} F_{3}$ & 78158.42 & 78162 & 1.26 \\
\hline $4 d^{3}\left({ }^{4} F\right) 5 p{ }^{5} D_{0}$ & 78568.37 & 78545 & 0.73 \\
\hline $4 d^{3}\left({ }^{4} F\right) 5 p{ }^{5} F_{1}$ & 78677.94 & 78618 & 1.09 \\
\hline $4 d^{3}\left({ }^{4} F\right) 5 p{ }^{5} G_{6}$ & 78689.51 & 78622 & 2.70 \\
\hline $4 d^{3}\left({ }^{4} F\right) 5 p{ }^{5} D_{1}$ & 78947.76 & 78952 & 0.92 \\
\hline $4 d^{3}\left({ }^{4} F\right) 5 p^{5} F_{2}$ & 79013.98 & 78952 & 1.15 \\
\hline $4 d^{3}\left({ }^{4} F\right) 5 p{ }^{5} D_{2}$ & 79467.93 & 79503 & 0.94 \\
\hline $4 d^{3}\left({ }^{4} F\right) 5 p^{5} F_{4}$ & 79497.10 & 79462 & 1.16 \\
\hline $4 d^{3}\left({ }^{4} F\right) 5 p^{5} D_{3}$ & 79508.33 & 79454 & 1.07 \\
\hline $4 d^{3}\left({ }^{4} F\right) 5 p{ }^{5} D_{4}$ & 80095.61 & 80061 & 0.89 \\
\hline $4 d^{3}\left({ }^{4} F\right) 5 p{ }^{5} F_{5}$ & 80343.19 & 80287 & 1.50 \\
\hline $4 d^{3}\left({ }^{4} F\right) 5 p{ }^{3} D_{3}$ & 80354.49 & 80423 & 1.12 \\
\hline $4 d^{3}\left({ }^{4} F\right) 5 p^{3} G_{3}$ & 81040.69 & 81047 & 1.49 \\
\hline $4 d^{3}\left({ }^{4} F\right) 5 p^{3} G_{4}$ & 82009.90 & 82017 & 1.45 \\
\hline $4 d^{3}\left({ }^{4} F\right) 5 p^{3} F_{2}$ & 82540.14 & 82570 & 1.83 \\
\hline $4 d^{3}\left({ }^{4} F\right) 5 p{ }^{3} G_{5}$ & 83147.76 & 83164 & 1.43 \\
\hline $4 d^{3}\left({ }^{4} F\right) 5 p^{3} F_{3}$ & 83584.53 & 83610 & 1.69 \\
\hline $4 d^{3}\left({ }^{2} \mathrm{P}\right) 5 \mathrm{p}{ }^{1} \mathrm{~S}_{0}$ & 84216.41 & 84307 & 3.92 \\
\hline $4 d^{3}\left({ }^{4} F\right) 5 p^{3} F_{4}$ & 84544.52 & 84542 & 1.57 \\
\hline $4 d^{3}\left({ }^{4} P\right) 5 p{ }^{5} P_{1}$ & 85308.94 & 85354 & 1.42 \\
\hline $4 d^{3}\left({ }^{4} P\right) 5 p{ }^{3} P_{2}$ & 85329.99 & 85406 & 2.44 \\
\hline $4 d^{3}\left({ }^{4} P\right) 5 p{ }^{5} D_{1}$ & 85683.11 & 85566 & 1.06 \\
\hline $4 d^{3}\left({ }^{2} G\right) 5 p{ }^{3} \mathrm{H}_{4}$ & 85896.20 & 86029 & 2.29 \\
\hline $4 d^{3}\left({ }^{4} P\right) 5 p{ }^{5} D_{0}$ & 86322.66 & 86395 & 3.18 \\
\hline $4 d^{3}\left({ }^{4} \mathrm{P}\right) 5 \mathrm{p}{ }^{5} \mathrm{P}_{2}$ & 86426.81 & 86318 & 0.83 \\
\hline $4 d^{3}\left({ }^{2} G\right) 5 p{ }^{3} \mathrm{H}_{5}$ & 86892.62 & 86989 & 2.04 \\
\hline $4 d^{3}\left({ }^{4} \mathrm{P}\right) 5 \mathrm{p}{ }^{5} \mathrm{P}_{3}$ & 87391.79 & 87252 & 0.80 \\
\hline $4 d^{3}\left({ }^{4} P\right) 5 p{ }^{5} D_{2}$ & 87473.30 & 87521 & 2.05 \\
\hline $4 d^{3}\left({ }^{4} P\right) 5 p{ }^{5} D_{3}$ & 87810.66 & 87822 & 1.88 \\
\hline $4 d^{3}\left({ }^{4} \mathrm{P}\right) 5 \mathrm{p}{ }^{3} \mathrm{P}_{1}$ & 87831.20 & 87945 & 1.91 \\
\hline $4 d^{3}\left({ }^{2} G\right) 5 p{ }^{3} H_{6}$ & 88441.64 & 88523 & 2.26 \\
\hline $4 d^{3}\left({ }^{2} G\right) 5 p{ }^{1} F_{3}$ & 88499.21 & 88597 & 1.00 \\
\hline $4 d^{3}\left({ }^{2} P\right) 5 p^{1} D_{2}$ & 88592.07 & 88603 & 1.70 \\
\hline $4 d^{3}\left({ }^{2} P\right) 5 p{ }^{3} P_{0}$ & 88669.74 & 88638 & 0.99 \\
\hline $4 d^{3}\left({ }^{4} P\right) 5 p{ }^{5} D_{4}$ & 89100.17 & 89160 & 2.31 \\
\hline $4 d^{3}\left({ }^{2} \mathrm{P}\right) 5 \mathrm{p}^{3} \mathrm{P}_{1}$ & 89139.81 & 89049 & 1.09 \\
\hline $4 d^{3}\left({ }^{2} G\right) 5 p^{3} F_{4}$ & 89503.85 & 89523 & 1.01 \\
\hline $4 d^{3}\left({ }^{2} G\right) 5 p{ }^{1} \mathrm{H}_{5}$ & 89689.91 & 89663 & 2.08 \\
\hline $4 d^{3}\left({ }^{4} P\right) 5 p{ }^{3} P_{0}$ & 89775.81 & 89671 & 1.94 \\
\hline $4 d^{3}\left({ }^{2} G\right) 5 p{ }^{3} G_{4}$ & 90255.05 & 90292 & 0.80 \\
\hline $4 d^{3}\left({ }^{2} P\right) 5 p^{3} D_{1}$ & 90301.83 & 90045 & 1.25 \\
\hline $4 d^{3}\left({ }^{2} G\right) 5 p{ }^{3} F_{2}$ & 90586.00 & 90688 & 1.26 \\
\hline $4 d^{3}\left({ }^{2} G\right) 5 p{ }^{3} G_{3}$ & 90588.46 & 90697 & 0.95 \\
\hline $4 d^{3}\left({ }^{2} \mathrm{P}\right) 5 \mathrm{p}^{3} \mathrm{P}_{2}$ & 90982.60 & 90984 & 1.10 \\
\hline $4 d^{3}\left({ }^{2} G\right) 5 p^{3} G_{5}$ & 91006.90 & 90990 & 0.91 \\
\hline $4 d^{3}\left({ }^{2} G\right) 5 p^{3} F_{3}$ & 91050.30 & 91159 & 1.20 \\
\hline $4 d^{3}\left({ }^{2} \mathrm{H}\right) 5 \mathrm{p}^{3} \mathrm{H}_{4}$ & 91387.50 & 91389 & 0.90 \\
\hline $4 d^{3}\left({ }^{2} P\right) 5 p{ }^{3} D_{2}$ & 91674.55 & 91562 & 1.20 \\
\hline $4 d^{3}\left({ }^{4} P\right) 5 p^{5} S_{2}$ & 92099.55 & 92222 & 1.78 \\
\hline $4 d^{3}\left({ }^{2} \mathrm{H}\right) 5 \mathrm{p}^{3} \mathrm{H}_{5}$ & 92254.52 & 92162 & 1.02 \\
\hline $4 d^{3}\left({ }^{2} \mathrm{H}\right) 5 \mathrm{p}^{3} \mathrm{H}_{6}$ & 92728.95 & 92619 & 1.00 \\
\hline
\end{tabular}


Table 2. (Continued.)

\begin{tabular}{|c|c|c|c|}
\hline Level & $\begin{array}{l}\mathrm{E}_{\exp }{ }^{\mathrm{a}} \\
\left(\mathrm{cm}^{-1}\right)\end{array}$ & $\begin{array}{l}E_{\text {calc }}{ }^{b} \\
\left(\mathrm{~cm}^{-1}\right)\end{array}$ & $\begin{array}{c}\text { Lifetime }^{\mathrm{b}} \\
\text { (ns) }\end{array}$ \\
\hline $4 d^{3}\left({ }^{2} P\right) 5 p{ }^{3} D_{3}$ & 92758.61 & 92667 & 0.98 \\
\hline $4 d^{3}\left({ }^{2} \mathrm{H}\right) 5 \mathrm{p}^{3} \mathrm{I}_{5}$ & 92884.18 & 92841 & 1.76 \\
\hline $4 d^{3}\left({ }^{2} G\right) 5 p{ }^{1} G_{4}$ & 93102.01 & 93198 & 1.05 \\
\hline $4 d^{3}\left({ }^{2} P\right) 5 p{ }^{3} S_{1}$ & 93222.37 & 92904 & 0.94 \\
\hline $4 d^{3}\left({ }^{2} H\right) 5 p{ }^{3} I_{6}$ & 93306.10 & 93224 & 2.20 \\
\hline $4 d^{3}\left({ }^{2} D\right) 5 p{ }^{3} F_{2}$ & 93642.52 & 93696 & 0.82 \\
\hline $4 d^{3}\left({ }^{2} \mathrm{D}\right) 5 \mathrm{p}{ }^{1} \mathrm{P}_{1}$ & 93709.46 & 93668 & 0.81 \\
\hline $4 d^{3}\left({ }^{2} H\right) 5 p{ }^{1} G_{4}$ & 94098.26 & 94258 & 1.35 \\
\hline $4 d^{3}\left({ }^{2} \mathrm{D}\right) 5 \mathrm{p}^{3} \mathrm{~F}_{3}$ & 94117.58 & 94132 & 0.80 \\
\hline $4 d^{3}\left({ }^{2} D\right) 5 p^{3} D_{1}$ & 94292.66 & 94396 & 0.88 \\
\hline $4 d^{3}\left({ }^{4} P\right) 5 p^{3} D_{2}$ & 94387.70 & 94479 & 0.93 \\
\hline $4 d^{3}\left({ }^{2} \mathrm{H}\right) 5 \mathrm{p}^{3} \mathrm{I}_{7}$ & 94424.07 & 94335 & 2.47 \\
\hline $4 d^{3}\left({ }^{4} P\right) 5 p^{3} D_{3}$ & 94676.73 & 94698 & 0.95 \\
\hline $4 d^{3}\left({ }^{2} D\right) 5 p{ }^{3} F_{4}$ & 94955.85 & 94999 & 0.88 \\
\hline $4 d^{3}\left({ }^{4} P\right) 5 p^{3} D_{1}$ & 95016.32 & 94975 & 0.88 \\
\hline $4 d^{3}\left({ }^{2} D\right) 5 p^{3} D_{2}$ & 95551.80 & 95534 & 0.84 \\
\hline $4 d^{3}\left({ }^{2} D\right) 5 p^{3} D_{3}$ & 95856.45 & 95884 & 1.09 \\
\hline $4 d^{3}\left({ }^{2} H\right) 5 p{ }^{3} G_{5}$ & 96285.38 & 96170 & 0.68 \\
\hline $4 d^{3}\left({ }^{2} \mathrm{D}\right) 5 \mathrm{p}^{3} \mathrm{P}_{2}$ & 96589.89 & 96592 & 0.90 \\
\hline $4 d^{3}\left({ }^{2} \mathrm{D}\right) 5 \mathrm{p}^{3} \mathrm{P}_{1}$ & 96736.45 & 96826 & 0.94 \\
\hline $4 d^{3}\left({ }^{2} H\right) 5 p{ }^{3} G_{3}$ & 96838.34 & 96850 & 0.79 \\
\hline $4 d^{3}\left({ }^{2} \mathrm{H}\right) 5 \mathrm{p}{ }^{1} \mathrm{I}_{6}$ & 96907.92 & 96762 & 1.14 \\
\hline $4 d^{3}\left({ }^{2} \mathrm{D}\right) 5 \mathrm{p}^{3} \mathrm{P}_{0}$ & 97135.60 & 97189 & 0.92 \\
\hline $4 d^{3}\left({ }^{2} H\right) 5 p^{3} G_{4}$ & 97184.77 & 97192 & 0.83 \\
\hline $4 d^{3}\left({ }^{2} \mathrm{H}\right) 5 \mathrm{p}{ }^{1} \mathrm{H}_{5}$ & 97709.08 & 97709 & 0.60 \\
\hline $4 d^{3}\left({ }^{2} \mathrm{D}\right) 5 \mathrm{p}{ }^{1} \mathrm{~F}_{3}$ & 98562.38 & 98589 & 0.68 \\
\hline $4 d^{3}\left({ }^{2} \mathrm{P}\right) 5 \mathrm{p}{ }^{1} \mathrm{P}_{1}$ & 99313.02 & 99336 & 0.94 \\
\hline $4 d^{3}\left({ }^{2} F\right) 5 p^{3} F_{2}$ & 99952.26 & 99960 & 0.74 \\
\hline $4 d^{3}\left({ }^{4} P\right) 5 p{ }^{3} S_{1}$ & 100184.65 & 100178 & 1.06 \\
\hline $4 d^{3}\left({ }^{2} D\right) 5 p{ }^{1} D_{2}$ & 100219.97 & 100213 & 1.24 \\
\hline $4 d^{3}\left({ }^{2} F\right) 5 p^{3} F_{3}$ & 100397.67 & 100389 & 1.28 \\
\hline $4 d^{3}\left({ }^{2} F\right) 5 p{ }^{3} F_{4}$ & 100858.67 & 100842 & 1.30 \\
\hline $4 d^{3}\left({ }^{2} F\right) 5 p{ }^{3} G_{3}$ & 102557.67 & 102504 & 1.60 \\
\hline $4 d^{3}\left({ }^{2} F\right) 5 p{ }^{3} G_{4}$ & 103276.74 & 103211 & 1.51 \\
\hline $4 d^{3}\left({ }^{2} F\right) 5 p{ }^{1} D_{2}$ & 103303.98 & 103501 & 1.14 \\
\hline $4 d^{3}\left({ }^{2} F\right) 5 p{ }^{3} G_{5}$ & 103621.4 & 103536 & 1.54 \\
\hline $4 d^{3}\left({ }^{2} F\right) 5 p{ }^{3} D_{3}$ & 103667.40 & 103744 & 1.10 \\
\hline $4 d^{3}\left({ }^{2} F\right) 5 p{ }^{3} D_{2}$ & 104511.12 & 104626 & 1.07 \\
\hline $4 d^{3}\left({ }^{2} F\right) 5 p^{3} D_{1}$ & 105041.26 & 105175 & 1.05 \\
\hline $4 d^{3}\left({ }^{2} F\right) 5 p{ }^{1} G_{4}$ & 106511.94 & 106530 & 1.15 \\
\hline $4 d^{3}\left({ }^{2} F\right) 5 p{ }^{1} F_{3}$ & 106803.63 & 106726 & 0.90 \\
\hline $4 d^{3}\left({ }^{2} D\right) 5 p^{3} D_{1}$ & 114014.74 & 114042 & 0.87 \\
\hline $4 d^{3}\left({ }^{2} D\right) 5 p^{3} D_{2}$ & 114083.06 & 114080 & 0.90 \\
\hline $4 d^{3}\left({ }^{2} D\right) 5 p^{3} D_{3}$ & 114591.26 & 114538 & 0.90 \\
\hline $4 d^{3}\left({ }^{2} D\right) 5 p^{3} F_{2}$ & 115794.02 & 115799 & 1.28 \\
\hline $4 d^{3}\left({ }^{2} D\right) 5 p^{3} F_{3}$ & 116497.95 & 116385 & 1.25 \\
\hline $4 d^{3}\left({ }^{2} D\right) 5 p^{3} F_{4}$ & 117287.80 & 117164 & 1.38 \\
\hline $4 d^{3}\left({ }^{2} D\right) 5 p{ }^{1} D_{2}$ & 117336.75 & 117392 & 0.95 \\
\hline $4 d^{3}\left({ }^{2} D\right) 5 p{ }^{3} P_{2}$ & 118451.23 & 118432 & 1.21 \\
\hline $4 d^{2}\left({ }^{3} F\right) 5 s 5 p^{5} G_{2}$ & 119170.3 & 119106 & 7.73 \\
\hline $4 d^{3}\left({ }^{2} D\right) 5 p^{3} P_{1}$ & 119206.22 & 119230 & 1.22 \\
\hline $4 d^{3}\left({ }^{2} D\right) 5 p{ }^{1} F_{3}$ & 119479.53 & 119402 & 1.01 \\
\hline $4 d^{3}\left({ }^{2} D\right) 5 p^{3} P_{0}$ & 119559.55 & 119602 & 1.22 \\
\hline $4 d^{2}\left({ }^{3} F\right) 5 s 5 p{ }^{5} G_{3}$ & 120064.7 & 120027 & 7.27 \\
\hline $4 d^{2}\left({ }^{3} F\right) 5 s 5 p^{5} G_{4}$ & 121118.4 & 121255 & 6.94 \\
\hline $4 d^{2}\left({ }^{3} F\right) 5 s 5 p{ }^{5} F_{1}$ & 121723.8 & 121620 & 0.90 \\
\hline $4 d^{2}\left({ }^{3} F\right) 5 s 5 p{ }^{5} F_{2}$ & 122229.55 & 122149 & 0.90 \\
\hline $4 d^{2}\left({ }^{3} F\right) 5 s 5 p{ }^{5} G_{5}$ & 122817.2 & 122755 & 6.82 \\
\hline
\end{tabular}

Table 2. (Continued.)

\begin{tabular}{|c|c|c|c|}
\hline Level & $\begin{array}{l}\mathrm{E}_{\exp }{ }^{\mathrm{a}} \\
\left(\mathrm{cm}^{-1}\right)\end{array}$ & $\begin{array}{l}E_{\text {calc }}{ }^{b} \\
\left(\mathrm{~cm}^{-1}\right)\end{array}$ & $\begin{array}{c}\text { Lifetime }^{\mathrm{b}} \\
\text { (ns) }\end{array}$ \\
\hline $4 d^{2}\left({ }^{3} F\right) 5 s 5 p{ }^{5} F_{3}$ & 123007.56 & 122959 & 0.90 \\
\hline $4 d^{2}\left({ }^{3} F\right) 5 s 5 p{ }^{5} F_{4}$ & 124005.8 & 123990 & 0.90 \\
\hline $4 d^{3}\left({ }^{2} D\right) 5 p{ }^{1} P_{1}$ & 124221.46 & 124295 & 0.91 \\
\hline $4 d^{2}\left({ }^{3} F\right) 5 s 5 p^{5} G_{6}$ & 124605.7 & 124526 & 8.13 \\
\hline $4 d^{2}\left({ }^{3} F\right) 5 s 5 p^{5} D_{0}$ & 124982.8 & 125009 & 0.59 \\
\hline $4 d^{2}\left({ }^{3} F\right) 5 s 5 p{ }^{5} D_{1}$ & 125107.68 & 125145 & 0.61 \\
\hline $4 d^{2}\left({ }^{3} F\right) 5 s 5 p{ }^{5} F_{5}$ & 125143.67 & 125160 & 0.90 \\
\hline $4 d^{2}\left({ }^{3} F\right) 5 s 5 p^{5} D_{2}$ & 125359.42 & 125422 & 0.63 \\
\hline $4 d^{2}\left({ }^{3} F\right) 5 s 5 p^{5} D_{3}$ & 125786.8 & 125890 & 0.66 \\
\hline $4 d^{2}\left({ }^{3} F\right) 5 s 5 p^{5} D_{4}$ & 126533.5 & 126667 & 0.64 \\
\hline $4 d^{2}\left({ }^{3} F\right) 5 s 5 p{ }^{3} F_{2}$ & 127336.03 & 127442 & 1.79 \\
\hline $4 d^{2}\left({ }^{3} F\right) 5 s 5 p{ }^{3} F_{3}$ & 127795.88 & 127844 & 1.52 \\
\hline $4 d^{2}\left({ }^{3} F\right) 5 s 5 p^{3} D_{2}$ & 129055.2 & 129157 & 1.05 \\
\hline $4 d^{2}\left({ }^{3} F\right) 5 s 5 p^{3} D_{1}$ & 129065.63 & 129235 & 1.11 \\
\hline $4 d^{2}\left({ }^{3} F\right) 5 s 5 p^{3} F_{4}$ & 129383.82 & 129439 & 1.54 \\
\hline $4 d^{2}\left({ }^{3} F\right) 5 s 5 p^{3} D_{3}$ & 129964.64 & 130072 & 1.10 \\
\hline $4 d^{2}\left({ }^{3} P\right) 5 s 5 p{ }^{5} S_{2}$ & 130073.7 & 130105 & 0.61 \\
\hline $4 d^{2}\left({ }^{3} F\right) 5 s 5 p^{3} G_{3}$ & 130453.9 & 130373 & 1.22 \\
\hline $4 d^{2}\left({ }^{3} F\right) 5 s 5 p^{3} G_{4}$ & 131570.80 & 131567 & 1.14 \\
\hline $4 d^{2}\left({ }^{3} P\right) 5 s 5 p{ }^{5} D_{1}$ & 131782.5 & 131744 & 2.35 \\
\hline $4 d^{2}\left({ }^{3} P\right) 5 s 5 p{ }^{3} S_{1}$ & 132164.6 & 132104 & 0.86 \\
\hline $4 d^{2}\left({ }^{3} P\right) 5 s 5 p{ }^{5} D_{2}$ & 132439.5 & 132402 & 2.08 \\
\hline $4 d^{2}\left({ }^{3} F\right) 5 s 5 p^{3} G_{5}$ & 132792.84 & 132832 & 1.13 \\
\hline $4 d^{2}\left({ }^{3} P\right) 5 s 5 p^{5} D_{3}$ & 133255.4 & 133143 & 1.48 \\
\hline $4 d^{2}\left({ }^{3} F\right) 5 s 5 p{ }^{1} D_{2}$ & 133422.2 & 133372 & 1.20 \\
\hline $4 d^{2}\left({ }^{3} F\right) 5 s 5 p{ }^{1} F_{3}$ & 133818.4 & 133760 & 1.44 \\
\hline $4 d^{2}\left({ }^{3} P\right) 5 s 5 p{ }^{5} D_{4}$ & 134502.10 & 134420 & 1.61 \\
\hline $4 d^{2}\left({ }^{3} P\right) 5 s 5 p{ }^{5} P_{2}$ & 134695.4 & 134824 & 0.89 \\
\hline $4 d^{2}\left({ }^{3} P\right) 5 s 5 p{ }^{5} P_{1}$ & 134844.9 & 134869 & 1.15 \\
\hline $4 d^{2}\left({ }^{1} \mathrm{D}\right) 5 \mathrm{~s} 5 \mathrm{p}{ }^{3} \mathrm{~F}_{2}$ & 135721.81 & 135589 & 0.68 \\
\hline $4 d^{2}\left({ }^{1} \mathrm{D}\right) 5 \mathrm{~s} 5 \mathrm{p}^{3} \mathrm{P}_{2}$ & 135963.7 & 135956 & 1.14 \\
\hline $4 d^{2}\left({ }^{3} P\right) 5 s 5 p{ }^{5} P_{3}$ & 136281.5 & 136182 & 0.68 \\
\hline $4 d^{2}\left({ }^{1} D\right) 5 s 5 p{ }^{3} P_{1}$ & 136300.2 & 136373 & 0.97 \\
\hline $4 d^{2}\left({ }^{1} \mathrm{D}\right) 5 \mathrm{~s} 5 \mathrm{p}^{3} \mathrm{~F}_{3}$ & 136402.5 & 136321 & 1.53 \\
\hline $4 d^{2}\left({ }^{3} F\right) 5 s 5 p{ }^{1} G_{4}$ & 136575.7 & 136451 & 0.78 \\
\hline $4 d^{2}\left({ }^{1} G\right) 5 s 5 p^{3} G_{4}$ & 137605.1 & 137524 & 0.70 \\
\hline $4 d^{2}\left({ }^{1} G\right) 5 s 5 p^{3} G_{3}$ & 137796.5 & 137777 & 0.72 \\
\hline $4 d^{2}\left({ }^{1} G\right) 5 s 5 p{ }^{3} G_{5}$ & 138344.9 & 138162 & 0.64 \\
\hline $4 d^{2}\left({ }^{1} \mathrm{D}\right) 5 \mathrm{~s} 5 \mathrm{p}{ }^{3} \mathrm{~F}_{4}$ & 138688.1 & 138672 & 0.70 \\
\hline $4 d^{2}\left({ }^{1} D\right) 5 s 5 p{ }^{3} D_{3}$ & 139243.0 & 139257 & 1.35 \\
\hline $4 d^{2}\left({ }^{1} G\right) 5 s 5 p{ }^{3} H_{4}$ & 141176.2 & 141222 & 1.68 \\
\hline $4 d^{2}\left({ }^{1} G\right) 5 s 5 p{ }^{3} H_{5}$ & 141967.4 & 142045 & 1.86 \\
\hline $4 d^{2}\left({ }^{3} P\right) 5 s 5 p^{3} D_{1}$ & 142845.9 & 142974 & 0.93 \\
\hline $4 d^{2}\left({ }^{1} G\right) 5 s 5 p{ }^{3} H_{6}$ & 142940.8 & 143056 & 1.90 \\
\hline
\end{tabular}


Table 3. Oscillator strengths and transition probabilities for strong Mo III lines $(\log g f>0.0)$.

\begin{tabular}{|c|c|c|c|c|c|c|c|c|}
\hline \multirow{2}{*}{$\begin{array}{c}\text { Wavelength }^{\mathrm{a}} \\
(\AA)\end{array}$} & \multicolumn{3}{|c|}{ Lower level $^{\mathrm{b}}$} & \multicolumn{3}{|c|}{ Upper level $^{\mathrm{b}}$} & \multirow[b]{2}{*}{$\log g f^{\mathrm{c}}$} & \multirow{2}{*}{$\begin{array}{l}g A^{\mathrm{c}} \\
\left(\mathrm{s}^{-1}\right)\end{array}$} \\
\hline & $\mathrm{E}\left(\mathrm{cm}^{-1}\right)$ & Parity & $\mathrm{J}$ & $\mathrm{E}\left(\mathrm{cm}^{-1}\right)$ & Parity & $\mathrm{J}$ & & \\
\hline 1081.558 & 50482 & (e) & 6.0 & 142941 & (o) & 6.0 & 0.05 & $6.44 \mathrm{E}+09$ \\
\hline 1092.163 & 34225 & (e) & 4.0 & 125787 & (o) & 3.0 & 0.08 & $6.70 \mathrm{E}+09$ \\
\hline 1094.044 & 35129 & (e) & 5.0 & 126534 & (o) & 4.0 & 0.24 & $9.77 \mathrm{E}+09$ \\
\hline 1110.936 & 35129 & (e) & 5.0 & 125144 & (o) & 5.0 & 0.32 & $1.13 \mathrm{E}+10$ \\
\hline 1113.829 & 34225 & (e) & 4.0 & 124006 & (o) & 4.0 & 0.17 & $7.97 \mathrm{E}+09$ \\
\hline 1115.077 & 46602 & (e) & 4.0 & 136282 & (o) & 3.0 & 0.09 & $6.63 \mathrm{E}+09$ \\
\hline 1118.290 & 46300 & (e) & 3.0 & 135722 & (o) & 2.0 & 0.04 & $5.86 \mathrm{E}+09$ \\
\hline 1133.082 & 49542 & (e) & 4.0 & 137797 & (o) & 3.0 & 0.01 & $5.30 \mathrm{E}+09$ \\
\hline 1138.132 & 50482 & (e) & 6.0 & 138345 & (o) & 5.0 & 0.39 & $1.27 \mathrm{E}+10$ \\
\hline 1165.084 & 46962 & (e) & 5.0 & 132793 & (o) & 5.0 & 0.05 & $5.53 \mathrm{E}+09$ \\
\hline 1266.159 & 13276 & (e) & 5.0 & 92255 & (o) & 5.0 & 0.15 & $5.88 \mathrm{E}+09$ \\
\hline 1267.142 & 13811 & (e) & 6.0 & 92729 & (o) & 6.0 & 0.28 & $8.00 \mathrm{E}+09$ \\
\hline 1278.095 & 16714 & (e) & 5.0 & 94956 & (o) & 4.0 & 0.12 & $5.40 \mathrm{E}+09$ \\
\hline 1278.394 & 1872 & (e) & 4.0 & 80096 & (o) & 4.0 & 0.19 & $6.31 \mathrm{E}+09$ \\
\hline 1282.865 & 20612 & (e) & 4.0 & 98562 & (o) & 3.0 & 0.17 & $6.02 \mathrm{E}+09$ \\
\hline 1286.413 & 19974 & (e) & 6.0 & 97709 & (o) & 5.0 & 0.43 & $1.08 \mathrm{E}+10$ \\
\hline 1295.409 & 13811 & (e) & 6.0 & 91007 & (o) & 5.0 & 0.18 & $6.08 \mathrm{E}+09$ \\
\hline 1299.046 & 13276 & (e) & 5.0 & 90255 & (o) & 4.0 & 0.05 & $4.47 E+09$ \\
\hline 1299.809 & 19974 & (e) & 6.0 & 96908 & (o) & 6.0 & 0.25 & $6.97 \mathrm{E}+09$ \\
\hline 1310.413 & 19974 & (e) & 6.0 & 96285 & (o) & 5.0 & 0.23 & $6.64 \mathrm{E}+09$ \\
\hline 1370.884 & 27007 & (e) & 3.0 & 99952 & (o) & 2.0 & 0.11 & $4.54 \mathrm{E}+09$ \\
\hline 1421.506 & 36164 & (e) & 4.0 & 106512 & (o) & 4.0 & 0.11 & $4.23 \mathrm{E}+09$ \\
\hline 1685.611 & 92255 & (o) & 5.0 & 151580 & (e) & 5.0 & 0.20 & $3.76 \mathrm{E}+09$ \\
\hline 1739.112 & 85896 & (o) & 4.0 & 143397 & (e) & 3.0 & 0.01 & $2.22 \mathrm{E}+09$ \\
\hline 1756.767 & 74725 & (o) & 3.0 & 131647 & (e) & 2.0 & 0.03 & $2.32 \mathrm{E}+09$ \\
\hline 1758.462 & 74725 & (o) & 3.0 & 131593 & (e) & 3.0 & 0.09 & $2.62 \mathrm{E}+09$ \\
\hline 1760.551 & 85896 & (o) & 4.0 & 142697 & (e) & 4.0 & 0.39 & $5.29 \mathrm{E}+09$ \\
\hline 1769.522 & 73853 & (o) & 2.0 & 130366 & (e) & 3.0 & 0.58 & $8.17 \mathrm{E}+09$ \\
\hline 1771.068 & 75817 & (o) & 4.0 & 132280 & (e) & 3.0 & 0.17 & $3.13 \mathrm{E}+09$ \\
\hline 1774.390 & 75817 & (o) & 4.0 & 132174 & (e) & 4.0 & 0.17 & $3.11 \mathrm{E}+09$ \\
\hline 1779.567 & 74725 & (o) & 3.0 & 130918 & (e) & 4.0 & 0.67 & $9.78 \mathrm{E}+09$ \\
\hline 1779.672 & 100398 & (o) & 3.0 & 156588 & (e) & 3.0 & 0.22 & $3.50 \mathrm{E}+09$ \\
\hline 1783.990 & 86893 & (o) & 5.0 & 142947 & (e) & 5.0 & 0.22 & $3.50 \mathrm{E}+09$ \\
\hline 1787.959 & 86893 & (o) & 5.0 & 142822 & (e) & 6.0 & 0.73 & $1.12 \mathrm{E}+10$ \\
\hline 1788.224 & 77113 & (o) & 5.0 & 133035 & (e) & 4.0 & 0.31 & $4.25 \mathrm{E}+09$ \\
\hline 1790.894 & 77113 & (o) & 5.0 & 132951 & (e) & 5.0 & 0.22 & $3.48 \mathrm{E}+09$ \\
\hline 1792.393 & 75817 & (o) & 4.0 & 131608 & (e) & 5.0 & 0.75 & $1.15 \mathrm{E}+10$ \\
\hline 1795.977 & 88442 & (o) & 6.0 & 144122 & (e) & 5.0 & 0.12 & $2.70 \mathrm{E}+09$ \\
\hline 1801.148 & 100859 & (o) & 4.0 & 156379 & (e) & 4.0 & 0.36 & $4.71 E+09$ \\
\hline 1801.682 & 92255 & (o) & 5.0 & 147758 & (e) & 6.0 & 0.34 & $4.50 \mathrm{E}+09$ \\
\hline 1801.880 & 92255 & (o) & 5.0 & 147752 & (e) & 5.0 & 0.24 & $3.55 \mathrm{E}+09$ \\
\hline 1803.661 & 76837 & (o) & 2.0 & 132280 & (e) & 3.0 & 0.03 & $2.19 \mathrm{E}+09$ \\
\hline 1805.453 & 88442 & (o) & 6.0 & 143829 & (e) & 6.0 & 0.49 & $6.31 \mathrm{E}+09$ \\
\hline 1807.635 & 78690 & (o) & 6.0 & 134010 & (e) & 6.0 & 0.44 & $5.65 \mathrm{E}+09$ \\
\hline 1807.955 & 77113 & (o) & 5.0 & 132424 & (e) & 6.0 & 0.83 & $1.38 \mathrm{E}+10$ \\
\hline 1809.786 & 92729 & (o) & 6.0 & 147984 & (e) & 6.0 & 0.32 & $4.29 \mathrm{E}+09$ \\
\hline 1810.468 & 92729 & (o) & 6.0 & 147963 & (e) & 7.0 & 0.07 & $2.40 \mathrm{E}+09$ \\
\hline 1815.078 & 89690 & (o) & 5.0 & 144784 & (e) & 6.0 & 0.58 & $7.75 \mathrm{E}+09$ \\
\hline 1815.120 & 78690 & (o) & 6.0 & 133782 & (e) & 5.0 & 0.33 & $4.33 \mathrm{E}+09$ \\
\hline 1815.310 & 88442 & (o) & 6.0 & 143529 & (e) & 7.0 & 0.81 & $1.29 \mathrm{E}+10$ \\
\hline 1822.281 & 78158 & (o) & 3.0 & 133035 & (e) & 4.0 & 0.24 & $3.50 \mathrm{E}+09$ \\
\hline 1822.356 & 92884 & (o) & 5.0 & 147758 & (e) & 6.0 & 0.67 & $9.34 \mathrm{E}+09$ \\
\hline 1824.171 & 92884 & (o) & 5.0 & 147704 & (e) & 4.0 & 0.09 & $2.47 \mathrm{E}+09$ \\
\hline 1827.558 & 94098 & (o) & 4.0 & 148816 & (e) & 5.0 & 0.08 & $2.39 \mathrm{E}+09$ \\
\hline 1829.585 & 93306 & (o) & 6.0 & 147963 & (e) & 7.0 & 0.80 & $1.26 \mathrm{E}+10$ \\
\hline 1829.887 & 78690 & (o) & 6.0 & 133338 & (e) & 7.0 & 0.95 & $1.77 \mathrm{E}+10$ \\
\hline 1834.584 & 78158 & (o) & 3.0 & 132667 & (e) & 3.0 & 0.01 & $2.05 \mathrm{E}+09$ \\
\hline 1836.682 & 93306 & (o) & 6.0 & 147752 & (e) & 5.0 & 0.24 & $3.41 \mathrm{E}+09$ \\
\hline 1838.390 & 97185 & (o) & 4.0 & 151580 & (e) & 5.0 & 0.03 & $2.09 \mathrm{E}+09$ \\
\hline
\end{tabular}


Table 3. (Continued.)

\begin{tabular}{|c|c|c|c|c|c|c|c|c|}
\hline \multirow{2}{*}{$\begin{array}{c}\text { Wavelength }^{\mathrm{a}} \\
(\AA)\end{array}$} & \multicolumn{3}{|c|}{ Lower level $^{\mathrm{b}}$} & \multicolumn{3}{|c|}{ Upper level $^{\mathrm{b}}$} & \multirow[b]{2}{*}{$\log g f^{\mathrm{c}}$} & \multirow{2}{*}{$\begin{array}{l}g A^{\mathrm{c}} \\
\left(\mathrm{s}^{-1}\right)\end{array}$} \\
\hline & $\mathrm{E}\left(\mathrm{cm}^{-1}\right)$ & Parity & $\mathrm{J}$ & $\mathrm{E}\left(\mathrm{cm}^{-1}\right)$ & Parity & $\mathrm{J}$ & & \\
\hline 1842.123 & 79497 & (o) & 4.0 & 133782 & (e) & 5.0 & 0.40 & $4.90 \mathrm{E}+09$ \\
\hline 1845.715 & 78158 & (o) & 3.0 & 132338 & (e) & 3.0 & 0.05 & $2.19 \mathrm{E}+09$ \\
\hline 1847.081 & 89690 & (o) & 5.0 & 143829 & (e) & 6.0 & 0.20 & $3.11 \mathrm{E}+09$ \\
\hline 1850.882 & 80343 & (o) & 5.0 & 134371 & (e) & 5.0 & 0.54 & $6.76 \mathrm{E}+09$ \\
\hline 1851.063 & 92255 & (o) & 5.0 & 146278 & (e) & 6.0 & 0.33 & $4.16 \mathrm{E}+09$ \\
\hline 1853.589 & 79497 & (o) & 4.0 & 133447 & (e) & 4.0 & 0.31 & $3.92 \mathrm{E}+09$ \\
\hline 1856.994 & 89100 & (o) & 4.0 & 142951 & (e) & 5.0 & 0.66 & $8.87 \mathrm{E}+09$ \\
\hline 1857.128 & 89100 & (o) & 4.0 & 142947 & (e) & 5.0 & 0.11 & $2.50 \mathrm{E}+09$ \\
\hline 1859.529 & 91007 & (o) & 5.0 & 144784 & (e) & 6.0 & 0.32 & $4.02 \mathrm{E}+09$ \\
\hline 1863.335 & 80343 & (o) & 5.0 & 134010 & (e) & 6.0 & 0.60 & $7.69 \mathrm{E}+09$ \\
\hline 1867.064 & 94424 & (o) & 7.0 & 147984 & (e) & 6.0 & 0.40 & $4.78 \mathrm{E}+09$ \\
\hline 1868.175 & 92729 & (o) & 6.0 & 146257 & (e) & 7.0 & 0.49 & $5.93 \mathrm{E}+09$ \\
\hline 1872.893 & 92884 & (o) & 5.0 & 146278 & (e) & 6.0 & 0.27 & $3.58 \mathrm{E}+09$ \\
\hline 1874.383 & 80096 & (o) & 4.0 & 133447 & (e) & 4.0 & 0.02 & $1.99 \mathrm{E}+09$ \\
\hline 1875.692 & 94118 & (o) & 3.0 & 147431 & (e) & 4.0 & 0.21 & $3.09 \mathrm{E}+09$ \\
\hline 1875.783 & 103277 & (o) & 4.0 & 156588 & (e) & 3.0 & 0.05 & $2.10 \mathrm{E}+09$ \\
\hline 1877.765 & 81041 & (o) & 3.0 & 134296 & (e) & 3.0 & 0.10 & $2.36 \mathrm{E}+09$ \\
\hline 1877.876 & 82010 & (o) & 4.0 & 135262 & (e) & 4.0 & 0.19 & $2.93 \mathrm{E}+09$ \\
\hline 1878.153 & 83148 & (o) & 5.0 & 136392 & (e) & 5.0 & 0.30 & $3.74 \mathrm{E}+09$ \\
\hline 1878.261 & 93102 & (o) & 4.0 & 146343 & (e) & 5.0 & 0.26 & $3.42 \mathrm{E}+09$ \\
\hline 1881.171 & 79508 & (o) & 3.0 & 132667 & (e) & 3.0 & 0.13 & $2.55 \mathrm{E}+09$ \\
\hline 1885.973 & 89690 & (o) & 5.0 & 142713 & (e) & 5.0 & 0.34 & $4.11 \mathrm{E}+09$ \\
\hline 1886.077 & 92884 & (o) & 5.0 & 145904 & (e) & 5.0 & 0.24 & $3.30 \mathrm{E}+09$ \\
\hline 1887.493 & 90588 & (o) & 3.0 & 143569 & (e) & 4.0 & 0.09 & $2.27 \mathrm{E}+09$ \\
\hline 1887.810 & 93306 & (o) & 6.0 & 146278 & (e) & 6.0 & 0.42 & $4.93 \mathrm{E}+09$ \\
\hline 1888.537 & 93306 & (o) & 6.0 & 146257 & (e) & 7.0 & 0.22 & $3.14 \mathrm{E}+09$ \\
\hline 1888.824 & 90255 & (o) & 4.0 & 143198 & (e) & 4.0 & 0.38 & $4.44 \mathrm{E}+09$ \\
\hline 1891.944 & 80096 & (o) & 4.0 & 132951 & (e) & 5.0 & 0.49 & $5.75 E+09$ \\
\hline 1892.802 & 83148 & (o) & 5.0 & 135980 & (e) & 6.0 & 0.76 & $1.07 \mathrm{E}+10$ \\
\hline 1893.133 & 91007 & (o) & 5.0 & 143829 & (e) & 6.0 & 0.30 & $3.68 \mathrm{E}+09$ \\
\hline 1893.640 & 90588 & (o) & 3.0 & 143397 & (e) & 3.0 & 0.07 & $2.13 \mathrm{E}+09$ \\
\hline 1894.035 & 80354 & (o) & 3.0 & 133152 & (e) & 3.0 & 0.12 & $2.47 \mathrm{E}+09$ \\
\hline 1894.313 & 82010 & (o) & 4.0 & 134800 & (e) & 5.0 & 0.71 & $9.58 \mathrm{E}+09$ \\
\hline 1895.468 & 103621 & (o) & 5.0 & 156379 & (e) & 4.0 & 0.16 & $2.69 \mathrm{E}+09$ \\
\hline 1896.304 & 91388 & (o) & 4.0 & 144122 & (e) & 5.0 & 0.18 & $2.79 \mathrm{E}+09$ \\
\hline 1897.184 & 83148 & (o) & 5.0 & 135857 & (e) & 4.0 & 0.19 & $2.83 \mathrm{E}+09$ \\
\hline 1897.588 & 81041 & (o) & 3.0 & 133739 & (e) & 4.0 & 0.65 & $8.27 \mathrm{E}+09$ \\
\hline 1898.774 & 79508 & (o) & 3.0 & 132174 & (e) & 4.0 & 0.48 & $5.55 \mathrm{E}+09$ \\
\hline 1899.149 & 82010 & (o) & 4.0 & 134665 & (e) & 3.0 & 0.13 & $2.51 \mathrm{E}+09$ \\
\hline 1899.458 & 91007 & (o) & 5.0 & 143654 & (e) & 5.0 & 0.38 & $4.46 \mathrm{E}+09$ \\
\hline 1901.914 & 79014 & (o) & 2.0 & 131593 & (e) & 3.0 & 0.33 & $3.92 \mathrm{E}+09$ \\
\hline 1902.156 & 82540 & (o) & 2.0 & 135112 & (e) & 2.0 & 0.18 & $2.76 \mathrm{E}+09$ \\
\hline 1903.938 & 81041 & (o) & 3.0 & 133563 & (e) & 2.0 & 0.03 & $1.97 \mathrm{E}+09$ \\
\hline 1906.291 & 90255 & (o) & 4.0 & 142713 & (e) & 5.0 & 0.28 & $3.52 \mathrm{E}+09$ \\
\hline 1912.105 & 83585 & (o) & 3.0 & 135883 & (e) & 3.0 & 0.37 & $4.27 E+09$ \\
\hline 1914.156 & 80096 & (o) & 4.0 & 132338 & (e) & 3.0 & 0.08 & $2.21 \mathrm{E}+09$ \\
\hline 1921.967 & 84545 & (o) & 4.0 & 136575 & (e) & 4.0 & 0.55 & $6.45 E+09$ \\
\hline 1925.304 & 91007 & (o) & 5.0 & 142947 & (e) & 5.0 & 0.06 & $2.07 \mathrm{E}+09$ \\
\hline 1926.479 & 96908 & (o) & 6.0 & 148816 & (e) & 5.0 & 0.43 & $4.81 \mathrm{E}+09$ \\
\hline 1928.750 & 84545 & (o) & 4.0 & 136392 & (e) & 5.0 & 0.56 & $6.50 \mathrm{E}+09$ \\
\hline 1929.270 & 94424 & (o) & 7.0 & 146257 & (e) & 7.0 & 0.56 & $6.55 \mathrm{E}+09$ \\
\hline 1930.278 & 94098 & (o) & 4.0 & 145904 & (e) & 5.0 & 0.15 & $2.49 \mathrm{E}+09$ \\
\hline 1932.167 & 82540 & (o) & 2.0 & 134296 & (e) & 3.0 & 0.24 & $3.11 \mathrm{E}+09$ \\
\hline 1934.708 & 96908 & (o) & 6.0 & 148595 & (e) & 7.0 & 0.89 & $1.40 \mathrm{E}+10$ \\
\hline 1935.096 & 83585 & (o) & 3.0 & 135262 & (e) & 4.0 & 0.33 & $3.79 \mathrm{E}+09$ \\
\hline 1945.802 & 92729 & (o) & 6.0 & 144122 & (e) & 5.0 & 0.17 & $2.59 \mathrm{E}+09$ \\
\hline 1959.453 & 106512 & (o) & 4.0 & 157547 & (e) & 3.0 & 0.15 & $2.43 \mathrm{E}+09$ \\
\hline 1968.516 & 92729 & (o) & 6.0 & 143529 & (e) & 7.0 & 0.33 & $3.69 \mathrm{E}+09$ \\
\hline 1970.716 & 106804 & (o) & 3.0 & 157547 & (e) & 3.0 & 0.07 & $1.99 \mathrm{E}+09$ \\
\hline
\end{tabular}


Table 3. (Continued.)

\begin{tabular}{|c|c|c|c|c|c|c|c|c|}
\hline \multirow{2}{*}{$\begin{array}{c}\text { Wavelength }^{\mathrm{a}} \\
(\AA)\end{array}$} & \multicolumn{3}{|c|}{ Lower level $^{b}$} & \multicolumn{3}{|c|}{ Upper level $^{\mathrm{b}}$} & \multirow[b]{2}{*}{$\log g f^{\mathrm{c}}$} & \multirow{2}{*}{$\begin{array}{l}g A^{\mathrm{c}} \\
\left(\mathrm{s}^{-1}\right)\end{array}$} \\
\hline & $\mathrm{E}\left(\mathrm{cm}^{-1}\right)$ & Parity & $\mathrm{J}$ & $\mathrm{E}\left(\mathrm{cm}^{-1}\right)$ & Parity & $\mathrm{J}$ & & \\
\hline 1977.561 & 97185 & (o) & 4.0 & 147752 & (e) & 5.0 & 0.07 & $2.01 \mathrm{E}+09$ \\
\hline 1983.340 & 94677 & (o) & 3.0 & 145097 & (e) & 4.0 & 0.33 & $3.58 \mathrm{E}+09$ \\
\hline 2074.234 & 97709 & (o) & 5.0 & 145904 & (e) & 5.0 & 0.09 & $1.92 \mathrm{E}+09$ \\
\hline 2113.644 & 49542 & (e) & 4.0 & 96838 & (o) & 3.0 & 0.03 & $1.62 \mathrm{E}+09$ \\
\hline 2133.072 & 50319 & (e) & 5.0 & 97185 & (o) & 4.0 & 0.20 & $2.32 \mathrm{E}+09$ \\
\hline 2170.584 & 33452 & (e) & 3.0 & 79508 & (o) & 3.0 & 0.06 & $1.63 \mathrm{E}+09$ \\
\hline 2179.381 & 34225 & (e) & 4.0 & 80096 & (o) & 4.0 & 0.02 & $1.48 \mathrm{E}+09$ \\
\hline 2182.544 & 50482 & (e) & 6.0 & 96285 & (o) & 5.0 & 0.06 & $1.58 \mathrm{E}+09$ \\
\hline 2184.304 & 46962 & (e) & 5.0 & 92729 & (o) & 6.0 & 0.09 & $1.72 \mathrm{E}+09$ \\
\hline 2190.445 & 43462 & (e) & 3.0 & 89100 & (o) & 4.0 & 0.23 & $2.35 \mathrm{E}+09$ \\
\hline 2211.028 & 35129 & (e) & 5.0 & 80343 & (o) & 5.0 & 0.26 & $2.47 \mathrm{E}+09$ \\
\hline 2214.400 & 42666 & (e) & 2.0 & 87811 & (o) & 3.0 & 0.10 & $1.72 \mathrm{E}+09$ \\
\hline 2216.611 & 72188 & (e) & 3.0 & 117288 & (o) & 4.0 & 0.36 & $3.11 \mathrm{E}+09$ \\
\hline 2224.649 & 58730 & (e) & 4.0 & 103667 & (o) & 3.0 & 0.01 & $1.37 \mathrm{E}+09$ \\
\hline 2226.929 & 58730 & (e) & 4.0 & 103621 & (o) & 5.0 & 0.43 & $3.63 \mathrm{E}+09$ \\
\hline 2252.421 & 58894 & (e) & 3.0 & 103277 & (o) & 4.0 & 0.30 & $2.63 \mathrm{E}+09$ \\
\hline 2253.197 & 35129 & (e) & 5.0 & 79497 & (o) & 4.0 & 0.18 & $1.97 \mathrm{E}+09$ \\
\hline 2257.204 & 46300 & (e) & 3.0 & 90588 & (o) & 3.0 & 0.05 & $1.49 \mathrm{E}+09$ \\
\hline 2264.742 & 72356 & (e) & 2.0 & 116498 & (o) & 3.0 & 0.22 & $2.14 \mathrm{E}+09$ \\
\hline 2269.714 & 46962 & (e) & 5.0 & 91007 & (o) & 5.0 & 0.22 & $2.13 \mathrm{E}+09$ \\
\hline 2275.001 & 50482 & (e) & 6.0 & 94424 & (o) & 7.0 & 0.62 & $5.40 \mathrm{E}+09$ \\
\hline 2275.488 & 34225 & (e) & 4.0 & 78158 & (o) & 3.0 & 0.05 & $1.44 \mathrm{E}+09$ \\
\hline 2275.637 & 43462 & (e) & 3.0 & 87392 & (o) & 3.0 & 0.06 & $1.45 \mathrm{E}+09$ \\
\hline 2289.202 & 49089 & (e) & 2.0 & 92759 & (o) & 3.0 & 0.03 & $1.36 \mathrm{E}+09$ \\
\hline 2290.062 & 46602 & (e) & 4.0 & 90255 & (o) & 4.0 & 0.09 & $1.55 \mathrm{E}+09$ \\
\hline 2294.974 & 35129 & (e) & 5.0 & 78690 & (o) & 6.0 & 0.58 & $4.80 \mathrm{E}+09$ \\
\hline 2296.561 & 51426 & (e) & 3.0 & 94956 & (o) & 4.0 & 0.27 & $2.32 \mathrm{E}+09$ \\
\hline 2298.245 & 59060 & (e) & 2.0 & 102558 & (o) & 3.0 & 0.14 & $1.74 \mathrm{E}+09$ \\
\hline 2306.494 & 49542 & (e) & 4.0 & 92884 & (o) & 5.0 & 0.05 & $1.41 \mathrm{E}+09$ \\
\hline 2325.556 & 50319 & (e) & 5.0 & 93306 & (o) & 6.0 & 0.51 & $3.98 \mathrm{E}+09$ \\
\hline 2330.945 & 34225 & (e) & 4.0 & 77113 & (o) & 5.0 & 0.40 & $3.09 \mathrm{E}+09$ \\
\hline 2332.694 & 54853 & (e) & 5.0 & 97709 & (o) & 5.0 & 0.03 & $1.31 \mathrm{E}+09$ \\
\hline 2349.913 & 46962 & (e) & 5.0 & 89504 & (o) & 4.0 & 0.02 & $1.26 \mathrm{E}+09$ \\
\hline 2353.747 & 64331 & (e) & 3.0 & 106804 & (o) & 3.0 & 0.16 & $1.72 \mathrm{E}+09$ \\
\hline 2357.584 & 72188 & (e) & 3.0 & 114591 & (o) & 3.0 & 0.22 & $2.00 \mathrm{E}+09$ \\
\hline 2359.758 & 33452 & (e) & 3.0 & 75817 & (o) & 4.0 & 0.26 & $2.20 \mathrm{E}+09$ \\
\hline 2366.290 & 50482 & (e) & 6.0 & 92729 & (o) & 6.0 & 0.28 & $2.28 \mathrm{E}+09$ \\
\hline 2370.025 & 64331 & (e) & 3.0 & 106512 & (o) & 4.0 & 0.19 & $1.83 \mathrm{E}+09$ \\
\hline 2372.982 & 58730 & (e) & 4.0 & 100859 & (o) & 4.0 & 0.29 & $2.28 \mathrm{E}+09$ \\
\hline 2377.137 & 54853 & (e) & 5.0 & 96908 & (o) & 6.0 & 0.49 & $3.63 \mathrm{E}+09$ \\
\hline 2383.876 & 50319 & (e) & 5.0 & 92255 & (o) & 5.0 & 0.24 & $2.02 \mathrm{E}+09$ \\
\hline 2384.649 & 77557 & (e) & 2.0 & 119480 & (o) & 3.0 & 0.18 & $1.75 \mathrm{E}+09$ \\
\hline 2386.965 & 32843 & (e) & 2.0 & 74725 & (o) & 3.0 & 0.13 & $1.57 \mathrm{E}+09$ \\
\hline 2408.682 & 58894 & (e) & 3.0 & 100398 & (o) & 3.0 & 0.10 & $1.46 \mathrm{E}+09$ \\
\hline 2410.094 & 46962 & (e) & 5.0 & 88442 & (o) & 6.0 & 0.33 & $2.43 \mathrm{E}+09$ \\
\hline 2474.252 & 52698 & (e) & 4.0 & 93102 & (o) & 4.0 & 0.12 & $1.44 \mathrm{E}+09$ \\
\hline 2481.192 & 46602 & (e) & 4.0 & 86893 & (o) & 5.0 & 0.22 & $1.79 \mathrm{E}+09$ \\
\hline 2487.664 & 52698 & (e) & 4.0 & 92884 & (o) & 5.0 & 0.00 & $1.09 \mathrm{E}+09$ \\
\hline 2490.018 & 49542 & (e) & 4.0 & 89690 & (o) & 5.0 & 0.00 & $1.08 \mathrm{E}+09$ \\
\hline 2506.187 & 44655 & (e) & 4.0 & 84545 & (o) & 4.0 & 0.22 & $1.78 \mathrm{E}+09$ \\
\hline 2524.709 & 46300 & (e) & 3.0 & 85896 & (o) & 4.0 & 0.19 & $1.63 \mathrm{E}+09$ \\
\hline 2547.336 & 54853 & (e) & 5.0 & 94098 & (o) & 4.0 & 0.04 & $1.14 \mathrm{E}+09$ \\
\hline 2597.134 & 44655 & (e) & 4.0 & 83148 & (o) & 5.0 & 0.18 & $1.50 \mathrm{E}+09$ \\
\hline 2633.565 & 50482 & (e) & 6.0 & 88442 & (o) & 6.0 & 0.11 & $1.25 \mathrm{E}+09$ \\
\hline
\end{tabular}

\footnotetext{
a Wavelengths deduced from experimental energies reported by Iglesias et al (1990).

${ }^{\mathrm{b}}$ Iglesias et al (1990).

This work.
} 


\section{References}

Baumann M, Liening H and Lindel H 1978 Phys. Lett. A 68319

Biémont E et al 2005 Proc. SPIE 5830221

Biémont E et al 2011 Mon. Not. R. Astron. Soc. 4143350

Campos J et al 2005 Mon. Not. R. Astron. Soc. 363905

Cowan R D 1981 The Theory of Atomic Structure and Spectra (Berkeley, CA: University of California Press)

Dickerman P J and Deuel R W 1964 J. Quant. Spectrosc. Radiat. Transfer 4807

Duquette D W, Salih S and Lawler J E 1981 Phys. Lett. A 83214

Fivet V et al 2009 Mon. Not. R. Astron. Soc. 3962124

Fraga S, Karwowski J and Saxena K M S 1976 Handbook of Atomic Data (Amsterdam: Elsevier)

Hannaford P and Lowe R M 1983 J. Phys. B: At. Mol. Phys. 164539

Hartman $\mathrm{H}$ et al 2010 Phys. Rev. A 82052512

Iglesias L, Cabeza M I and Kaufman V 1990 J. Res. Natl Inst. Stand. Technol. 95647

Iglesias L, Cabeza M I, Rico F R and Kaufman V 1988 Phys. Scr. 37855

Jiang L Y et al 2012 Eur. Phys. J. D 66176

Jiang L Y et al 2013 J. Opt. Soc. Am. B 30489

Kwiatkowski M, Micali G, Werner K and Zimmermann P 1981 Phys. Lett. A 85273

Lipschultz B et al 2006 Phys. Plasmas 13056117

Liu Z X et al 2013 Nucl. Fusion 53073041

Lundberg H et al 2010 J. Phys. B: At. Mol. Opt. Phys. 43085004

Malcheva G et al 2006 Mon. Not. R. Astron. Soc. 367754
Malcheva G et al 2011 Mon. Not. R. Astron. Soc. 4121823

Nilsson H et al 2010 Astron. Astrophys. 511 A16

Palmeri P and Wyart J-F 1998 Phys. Scr. 58445

Palmeri P et al 2007 Mon. Not. R. Astron. Soc. 37463

Palmeri P et al 2009 J. Phys. B: At. Mol. Opt. Phys. 42165005

Peterson R C 2011 Astrophys. J 74221

Peterson R C 2013 Astrophys. J. Lett. 768 L13

Quinet P 2002 J. Phys. B: At. Mol. Opt. Phys. 3519

Quinet P et al 1999 Mon. Not. R. Astron. Soc. 307934

Quinet $\mathrm{P}$ et al 2002 J. Alloys Compd. 344255

Quinet P et al 2011 J. Electron Spectrosc. Relat. Phenom. 184174

Quinet $\mathrm{P}$ et al 2012 Astron. Astrophys. 537 A74

Salih S and Lawler J E 1983 Phys. Rev. A 283653

Schnehage S E, Danzmann K, Künnemeyer R and Kock M 1983 J. Quant. Spectrosc. Radiat. Transfer 29507

Sikström et al 2001 J. Phys. B: At. Mol. Opt. Phys. 34477

Whaling W 1984 J. Quant. Spectrosc. Radiat. Transfer 3269

Whaling W 1986 J. Quant. Spectrosc. Radiat. Transfer 36491

Whaling W and Brault J W 1988 Phys. Scr. 38707

Xu H L et al 2006 Astron. Astrophys. 452357

Zhang Y et al 2008 Phys. Rev. A 78022505

Zhang W et al 2009 Eur. Phys. J. D 551

Zhang W et al 2010 J. Phys. B: At. Mol. Opt. Phys. 43205005

Zhang W, Palmeri P and Quinet P 2013 Phys. Scr. 88065302

Zhang W, Palmeri P, Quinet P and Biémont E 2013 Astron. Astrophys. 551 A136

Zhang W, Palmeri P and Quinet P 2014 Eur. Phys. J. D 68104 


\section{QUERY FORM}

Journal: Physica Scripta

Author: P Quinet

TITLE: Atomic structure, radiative lifetime and oscillator strength calculations in doubly ionized molybdenum (Mo III)

ArTiCle ID: ps504644

The layout of this article has not yet been finalized. Therefore this proof may contain columns that are not fully balanced/ matched or overlapping text in inline equations; these issues will be resolved once the final corrections have been incorporated. 